\title{
Predictive and Prognostic Value of Heart Rate Variability Analysis in Early Bedside Diagnosis and Management of COVID-19 Patients
}

\author{
Nabil A Mageed* and Ibrahim I Abd El Baser \\ Department of Anesthesia and Surgical Intensive Care, Mansoura University, Egypt
}

*Corresponding author: Nabil A Mageed, Department of Anesthesia and Surgical Intensive Care, Mansoura University, Egypt.

Received Date: April 09, 2020

Published Date: April 17, 2020

\begin{abstract}
Viral infection, sepsis and multiple organ dysfunction are the most common causes of death in critically ill patients. Early diagnosis and early management are the key factor in improving outcome in sepsis due to viral infection, so, there is need to develop more rapid, sensitive, and specific diagnostic strategies that allow early intervention to improve patient outcome. Heart rate variability (HRV) has been documented to be both altered in the presence of systemic infection, and correlated with its severity. This aim of this article is to review the HRV analysis and its value in detection early diagnosis of viral infection including COVID-19 patients. The prognostic value of clinical bedside application of HRV in the management and treatment viral infection and sepsis are highlighted in this review. The overall HRV decreased with lower low frequency component has been shown to be consistently correlated with the presence and severity of viral infection. HRV monitoring may enhance the early diagnosis, prognosis and early initiation of treatment of viral infectionCOVID-19. Bedside wearable devices that are based on photoplethysmography such as watch-like devices and mobile phone apps may be used as a useful non-invasive tool for early diagnosis of COVID -19 by analyzing the HRV.
\end{abstract}

Keywords: Heart rate variability; COVID-19; Early; Diagnosis; Predictive

\section{Introduction}

Viral infection has been demonstrated to be the most common cause of morbidity and mortality, particularly in immunecompromised patients. In critically ill patients who are admitted to intensive care unit (ICU), septicemia and organ dysfunction represent a high mortality rate ranging from $10 \%$ to $59 \%$ for all ICU admissions [1,2,3]. Viral infection including Coronavirus disease 2019 (COVID-19) (also named SARS-CoV-2) increases capillary permeability resulting in internal hemorrhage and multiorgan dysfunction. The vascular permeability syndrome has been one of greatest challenges since dengue shock syndrome was first described $[4,5]$.

Understanding the harmony of the host response to septicemia and multiple organ dysfunction remains defective despite of the full knowledge of the involved receptor levels, mechanisms, mediators, gene activation and pathways. The early goal-directed therapy in patients with septic shock secondary to sever viral infection includes early diagnosis, aggressive resuscitation, maintenance of adequate tissue perfusion and oxygen delivery and starting the appropriate antiviral drug [6,7]. Kumar A, et al. [8] found that the delay for each hour in the initiation of the appropriate anti-viral therapy was associated with $7.6 \%$ decrease in the mean survival rate. The common causes of delayed diagnosis of viral infection with subsequent rapid deterioration, organ dysfunction and admission to ICU are waiting the onset of fever and the occurrence of changes in the vital signs. Early, rapid management and resuscitation is the cornerstone in improving the outcome in septic shock due to viral infection, for this reason, there is urgent need for development of new methods and techniques that allow more sensitive, specific and rapid diagnosis that could be complementary or superior to the existing conventional methods.

The biomarkers and serological studies allow only early detection of severe viral infection, but they are of limited use because of the poor diagnostic capability and the long time they take to get the results. The response of the host to viral infection includes a 
dynamic interaction between organs, cells, mediators, and genes resulting in an increase in a complex non-linear system $[9,10]$. The approach of the analysis this complex system by interval-in-time or variation of pattern over time is defined as variability analysis [11]. Recording heart rate variation over time intervals may be analysed with several mathematical techniques. Heart rate variability (HRV) analysis has several advantages as it's, a non-invasive, easy to apply, good reproducibility and have a useful role in the diagnosis of autonomic dysfunction and other pathological conditions. Heart rate variability has been demonstrated to be changed in response to systemic infection with strong correlation between its degree and the severity of infection. The aim of this article is to review the value of the use of HRV monitoring as a predictive for early diagnosis of viral infection including COVID-19. The values of clinical bedside application of HRV in the management and treatment viral infection and sepsis are highlighted in this review.

\section{Coronavirus Disease 2019 (COVID-19) (SARS- CoV-2)}

In the last months of 2019 year, outbreaks of cases with bronchopneumonia with no obvious cause emerged in Wuhan, Hubei Province, China [12]. A novel Corona virus named COVID-19 was considered to the cause of pneumonia [13]. Every day, thousands of patients with severe viral infection have died all over the world due unavailability of the specific antiviral drugs.

\section{Clinical Presentation and Diagnosis}

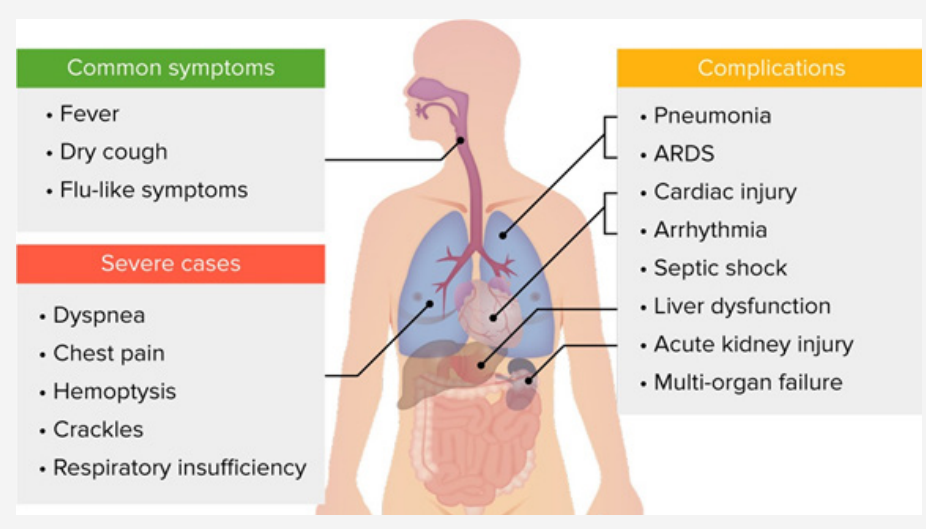

Figure 1: The clinical presentation of COVID-19.

Dry cough, rise of body temperature, easy fatigability and myalgia are the most common presentations. Less frequent symptoms are headache, productive cough, diarrhea and hemoptysis. More than $50 \%$ of infected patients develops dyspnea [14]. On performing complete blood count, the white blood cell count is either normal or low and lymphocyte count is reduced (lymphopenia) in 65\% of cases [14]. The classic findings on computed tomography (CT) in patients who are admitted to ICU are bilateral multiple consolidations that are lobular and sub segmental $[13,14]$. The clinical presentation of COVID-19 is illustrated in Figure 1.

The diagnosis of COVID-19 is based on the clinical presentation, radiologic imaging (chest X ray and high-resolution chest CT) [15] and detection the virus by serologic studies by RT-PCR [16] and CRISPR/Cas13 System [17].

\section{Pathogenesis of COVID-19}

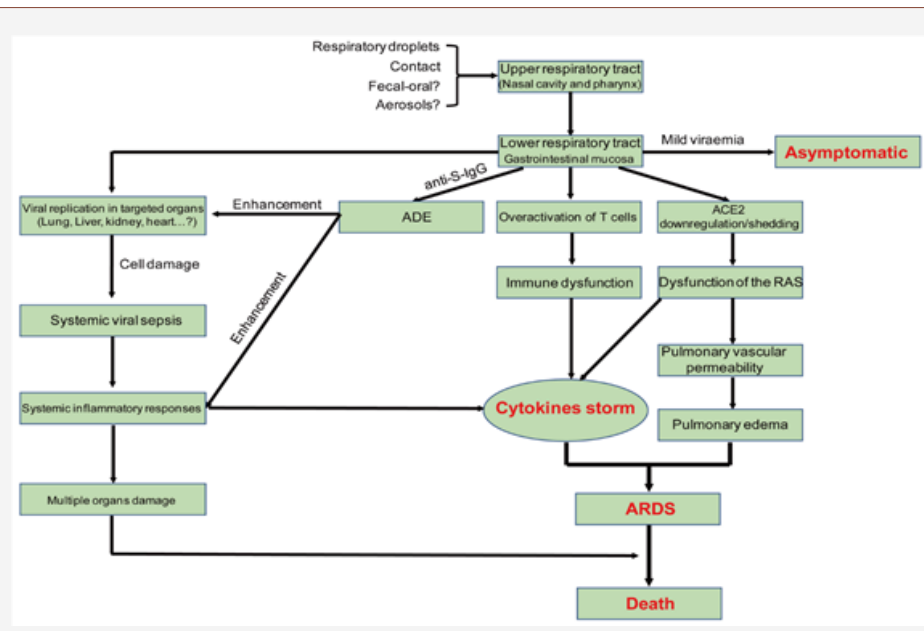

Figure 2: Shows the pathogenesis of SARS-CoV-2 infection.

Antibody-dependent enhancement (ADE); ACE2: angiotensin-converting enzyme 2; RAS: renin-angiotensin system; ARDS: acute respiratory distress syndrome [18]. 
The main routes of transmission of SARS-CoV-2 are droplet infection via respiratory tract, direct contact with the patient and through the fecal-oral route. The epithelium of upper respiratory tract mucosa is the initial site of viral replication with subsequent multiplication of the virus in the lung and gastrointestinal mucosa, resulting in mild viremia. Few patients remain asymptomatic at this stage, but some patients may show non-respiratory symptoms such as hepatic, cardiac, and renal failure with the development of multiple organ failure. The pathogenesis of SARS-CoV-2 host infection is illustrated in Figure 2.

\section{Severe Acute Respiratory Syndrome (SARS)}

SARS is a critical, severe life-threatening lung injury that interferes with pulmonary oxygenation and requires mechanical ventilation as the histopathological findings also confirms SARS [19] The occurrence of SARS is closely related to genetic susceptibility and the liberal release of inflammatory cytokines. Greater than 40 susceptible genes including ACE2, tumour necrosis factor (TNF), interleukin IL-6, IL-8, and IL0, and vascular endothelial growth factor are involved in the pathogenesis and outcome of SARS [20].

\section{Heart Rate Variability (HRV)}

\section{Pathophysiology}

Heart rate (HR) is a dynamic signal that varies with time. The variability in heart rate may indicate the presence of current pathologic condition. Heat rate variability (HRV) which is defined as the variation of the period between consecutive heartbeats over time, is mainly dependent on the extra cardiac regulation of the HR. HRV is a physiological phenomenon depending on factors modulating the cardiac activity produced by the autonomic nervous system [21]. Thus, HRV is a non-invasive and indirect assessment of the control of autonomic nervous system on cardiac function and provides an idea about the balance in the activity between the sympathetic and parasympathetic nervous systems. HRV shows that the structure generating the signal is not only simply linear, but also involves nonlinear contributions [22].

The parasympathetic control of HR is regulated through the release of acetylcholine by the vagus nerve. Muscarinic acetylcholine receptors respond to this release mostly by increasing the K2+ conductance in cell membrane. The sympathetic control of HR is mediated by the release of epinephrine and norepinephrine [23]. Rhythms from sympathetic and parasympathetic autonomic activity contribute independently on the modification of QRS complex intervals of $\mathrm{HR}$ in the electrocardiogram (ECG), at determined frequencies. The variation in RR interval is a normal finding during resting conditions represent beat-by-beat variations in cardiac autonomic inputs [24].

Alteration in HRV is closely related to the presence of illness and pathologic condition, and the degree of HRV alteration is an indicator of the severity of the current disease. A high degree of variability in HR means a healthy person with a good functioning autonomic nervous system. A low HRV indicates an abnormal autonomic system, HRV have been reported in a variety of medical conditions including myocardial infarction, diabetes, the metabolic syndrome, end-stage renal disease, chronic liver disease, hypoxic lung disease, congestive heart failure, and hypertension [25-29].

Altered HRV and autonomic dysfunction have been previously reported with viral infection. Carter, et al. [30] have been previously found that the cardiac parasympathetic activity (high frequency component) is responsible for the majority of the HRV reduction in patients with dengue viral infection [30]. Lebech AM, et al. [31] and Galdino GAM, et al. [32] have been suggested that the HIV and AIDS patients have abnormal HRV and the use of anti-viral therapy modifies the HRV.

\section{Techniques of HRV analysis}

There are several methods for analysis and evaluation of HRV alteration or altered patterns of heart rate change over time. The conventional method of assessment of HRV is based on calculating certain indices of statistical analytic tests on RR intervals (means and variance). The average HR, standard deviation of NN intervals [SDNN] and the root mean square of successive heartbeat interval differences [RMSSDs] are the most used indices. All the indices of time domain measure are liable to be influenced by outliers and artifacts, and these measures for this reason need data from which ectopic beats and artifacts have been accurately excluded [33]. Geometrical methods present RR intervals in geometric patterns and triangular index, Poincare plots have been used, but it is poorly sensitive to ectopic beats and artifacts [34].

Analysis of frequency domain is a unique and non-invasive method for obtaining a more accurate evaluation of autonomic activity and function. Depending on Fourier theory, any time series may be considered as a sum of sinusoidal oscillations with distinct frequencies. A time domain analysis can be converted to a frequency domain analysis by the fast Fourier transform (FFT) or the discrete Fourier transform, which can measure the spectral content of the signal in well-defined ranges of frequencies [35]. Techniques like wavelet analysis are referred to as 'time-frequency domain analyses'. High frequency [HF] component is far better understood than that of the lower frequency [LF] components. Respiratory pattern also can significantly affect HF power [36]. LF power is more complex as it is affected and controlled by both sympathetic and parasympathetic nervous system beside the other factors such as baroreceptor activity. Therefore, LF analysis by itself cannot afford an accurate delineation of the level of sympathetic activation [36].

Nonlinear dynamics and chaos theory can measure the dynamics of HR variation. Approximate entropy [ApEn] is a parameter and measure that can estimate the predictability or regularity of timeseries data [37]. The detrended fluctuation analysis (DFA) is a method of measurement which determine the presence or absence of fractal correlation properties and has been validated for timeseries data [38]. 


\section{The Benefits of HRV Analysis in COVID-19 Patients}

\section{Early diagnosis}

Analysis of HRV have been demonstrated to be useful for early diagnosis of infection, especially in neonates and infants. Kovatchev BP, et al. [39] have presented the sample asymmetry analysis (SAA) and showed its benefit for analysing heart rate characteristics [HRC] occurring in the early stage of neonatal sepsis and multiple organ dysfunction on 158 neonates and infants admitted to the intensive care unit. In their study, infants who suffered from sepsis when compared to normal healthy infants, had similar sample asymmetry in health, and elevated values before sepsis. They concluded that measurement of HRV was useful in predicting the early diagnosis of the onset of sepsis.

Griffin MP, et al. [40] and Moorman JR, et al. [41] introduced a novel HRC to analyse HRV in high risk neonates and infants for developing sepsis. They found that, the abnormal HRC with decreased HRV and temporary decelerations occurred just before the onset and diagnosis of sepsis in neonates and infants. Griffin MP, et al. [40] demonstrated abnormalities in HRV that were observed about 12 to 24 hours just before the onset, the clinical and laboratory diagnosis of sepsis. The traditional diagnosis of sepsis depends on the presence of fever, tachycardia and positive blood culture. Also, Cao H, et al. [42] measured the degree of nonstationarity in the heart rate signal to predict neonatal sepsis and they reported abnormal HRC of decreased HRV and temporary decelerations 12 to 24 hours just before the onset of neonatal sepsis.

\section{Prognosis of patient outcome}

Analysis of HRV may give us a valuable prognostic tool of infection in the critically ill patients. The degree and severity of the fluctuation in HRV may be correlated well with severity of the disease and may indicate high mortality rate and poor outcome of sepsis and viral infection. The frequency domain Fourier spectral analysis has been frequently used for prognosis and prediction the outcome of critically ill patients admitted to ICU [43]. Yien HW, et al. [44] reported that in ICU patients, a progressive decrease of LF and very LF components of HRV were demonstrated to be a significant marker of high risk of morbidity and mortality with poor outcome depending on trend changes in the LF and very LF components and correlated positively with Acute Physiological and Chronic Health Evaluation (APACHE) II scores.

Also, Soriano F, et al. [45], reported a significant correlation between LF power and mortality in critically ill patients admitted to ICU due to septic shock. Piepoli M, et al. [46] analyzed HRV in twelve critically ill patients during septic shock and during recovery from it. They reported ten patients who recovered from infection with normalization of LF component of HRV. Pontet J, et al. [47] used the power spectral analysis of HRV as an early indicator of septic shock and multiple organ dysfunction in septicemic patients. They demonstrated that HF power was significantly decreased in the studied patients.
Barnaby D, et al. [48] found that septicemia was the main illness leading to a reduction in the $\mathrm{LF} / \mathrm{HF}$ ratio $(\mathrm{LF} / \mathrm{HF}<1)$ and that a decreased LF/HF ratio correlated with a high risk of mortality. HRV was assessed in in septic shocked patients by Chen WL and Kuo CD [49] and found Normalized LF component and LF/HF components ratio were reduced.

\section{Bedside HRV analysis}

Most of the studies report HRV measurements to diagnose early sepsis 12 to 24 hours prior to traditional clinical methods [40-42]. However, the study by Kovatchev BP, et al. [39], the only one study reported observable changes in HRV as early as 3 to 4 days before the onset of sepsis. Ahmad S, et al. [50] studied the usefulness of HRV analysis for the early diagnosis of sepsis in adult bone marrow transplant patients for an average of 12-17 days and demonstrated satisfactory results including the specificity and sensitivity of frequent analysis of HRV metrics for the early detection of sepsis in adults.

Electrocardiography (ECG) is the traditional method of HRV analysis conducted by medical personnel's. Wearable devices for assessment of HRV may be a simple, low cost and convenient alternative. Wearable devices are reliable instruments for analysis of HRV in static posture. Nowadays, different wearable devices have been used as a bedside non-invasive test to evaluate HRV.They based on photoplethysmography (PPG). PPG is an optical method that detects cardiac beats by analyzing changes in light absorption and the color of skin. The PPG sensor sense the alteration in intensity of light via transmission through or reflection from the tissue. The variations in the light intensity are related to changes in the blood perfusion of the tissue, and based on these changes, heart-related information can be retrieved [51]. The most popular ones are the watch-like devices [52] and mobile phone applications, many smartphones have high speed data transmission capabilities like 3G, WiFi and external sensors connectivity via Bluetooth [53]. HRV indices that are derived from the Apple Watch RR interval series are correlated with changes caused by mental stress. Hernando D, et al. [52] found a significant decrease of HF power as well as RMSSD during mental stress HRV with respect to relax derived from Apple Watch.

\section{Conclusion}

The overall HRV decreased with lower Low frequency component has been shown to consistently correlate with the presence and severity of viral infection. HRV monitoring may enhance the early diagnosis, prognosis and initiation of treatment of viral infection in critically ill patients including COVID-19. Bedside wearable devices based on photoplethysmography such as watch-like devices and mobile phone application may be used as useful non-invasive tool for early diagnosis of COVID -19 through evaluating the HRV.

\section{Acknowledgement}

None. 


\section{Conflict of Interest}

No conflict of interest.

\section{References}

1. Billman GE (2011) Heart rate variability-a historical perspective. Front Physiol 2: 86.

2. Billman GE (2013) The effect of heart rate on the heart rate variability response to autonomic interventions. Front Physiol 4: 222.

3. Singer M, Deutschman CS, Seymour CW, Shankar Hari M, Annane D, et al. (2016) The Third International Consensus Definitions for sepsis and septic shock (Sepsis-3) JAMA 315(8): 801-810.

4. Cohen WL, Kuo CD (2007) Characteristics of heart rate variability can predict impending septic shock in emergency department patients with sepsis. Acad Emerg Med 14(5): 392-397.

5. Halstead SB, Nimmannitya S, Cohen SN (1970) Observations related to pathogenesis of dengue hemorrhagic fever. IV. Relation of disease severity to antibody response and virus recovered. Yale J Biol Med 42(5): 311-328.

6. Rivers EP, Nguyen B, Havstad S, Ressler J, Muzzin A, et al. (2001) Early goal-directed therapy in the treatment of severe sepsis and septic shock. N Eng J Med 345(19): 1368-1377.

7. Otero RM, Nguyen HB, Huang DT, Gaieski DF, Goyal M, et al. (2006) Early goal-directed therapy in severe sepsis and septic shock revisited: concepts, controversies, and contemporary findings. Chest 130(5):15791595.

8. Kumar A, Roberts D, Wood KE, Light B, Parrillo JE, et al. (2006) Duration of hypotension before initiation of effective antimicrobial therapy is the critical determinant of survival in human septic shock. Crit Care Med 34(6): 1589-1596.

9. Seely AJE, Christou NV (2000) Multiple organ dysfunction syndrome: exploring the paradigm of complex nonlinear systems. Crit Care Med 28(7): 2193-2200.

10. Seely AJE, Macklem PT (2004) Complex systems and the technology of variability analysis. Crit Care 8(6): R367-R384.

11. Stein PK, Kleiger RE (1999) Insights from the study of heart rate variability. Annu Rev Med 50:249-261.

12. Li, Q, Guan X, Wu P, Wang X, Zhou L, et al. (2020) Early Transmission Dynamics in Wuhan, China, of Novel Coronavirus-Infected Pneumonia. N Engl J Med 382(13): 1199-1207.

13. Zhu N, Zhang D, Wang W, Li X, Yang B, et al. (2020) A Novel Coronavirus from Patients with Pneumonia in China, 2019. N Engl J Med 382(8):727733.

14. Huang C, Wang Y, Li X, Ren L, Zhao J, et al. (2020) Clinical features of patients infected with 2019 novel coronavirus in Wuhan, China. Lancet 395(10223): 497-506.

15. Kanne JP (2020) Chest CT Findings in 2019 Novel Coronavirus (2019$\mathrm{nCoV}$ ) Infections from Wuhan, China: Key Points for the Radiologist. Radiology 295(1): 16-17.

16. Vashist SK (2020) In Vitro Diagnostic Assays for COVID-19: Recent Advances and Emerging Trends. Diagnostics 10: 202.

17. Myhrvold C, Freije CA, Gootenberg JS, Abudayyeh 00, Metsky HC, et al. (2018) Field-deployable viral diagnostics using CRISPR-Cas13. Science 360(6387): 444-448

18. Jin Y, Yang H, Ji W, Wu W, Chen S, et al. (2020) Review: Virology, Epidemiology, Pathogenesis, and Control of COVID-19. Viruses 12(4): 372.

19. Ng DL, Al Hosani F, Keating MK, Gerber SI, Jones TL, et al. (2016) Clinicopathologic, Immunohistochemical, and Ultrastructural Findings of a Fatal Case of Middle East Respiratory Syndrome Coronavirus Infection in the United Arab Emirates, April 2014. Am J Pathol 186(3): 652-658
20. Thompson BT, Chambers RC, Liu KD (2017) Acute Respiratory Distress Syndrome. N Engl J Med 377: 562-572.

21. Saul JP(1990) Beat-to-beat variations of heart rate reflect modulation of cardiac autonomic outflow. News Physiol Sci 5: 32-33.

22. Kleiger RE, Bigger JT, Bosner MS, Chung MK, Cook JR, et al. (1991) Stability over time of variables measuring heart rate variability in normal subjects. Am J Cardiol 68(6): 626-630.

23. Kienzle MG, Ferguson DW, Birkett CL, Myers GA, Berg WJ, et al. (1992) Clinical, hemodynamic and sympathetic neural correlates of heart rate variability in congestive heart failure. Am J Cardiol 69(8): 761-767.

24. Hayano J, Yamada A, Mukai S, Sakakibara Y, Yamada M, et al. (1991) Severity of coronary atherosclerosis correlates with the respiratory component of heart rate variability. Am Heart J 121(4 Pt 1): 1070-1079.

25. Ahern DK, Gorkin L, Anderson JL, Tierney C, Hallstrom A, et al. (1990) Biobehavioral variables and mortality or cardiac arrest in the cardiac arrhythmia pilot study (CAPS). Am J Cardiol 66(1): 59-62.

26. Frasure Smith N, Lespérance F, Talajic M (1995) Depression and 18-month prognosis after myocardial infarction. Circulation 91(4): 9991005 .

27. Ponikowski P, Anker SD, Chua TP, Szelemej R, Piepoli M, et al. (1997) Depressed heart rate variability as an independent predictor of death in chronic congestive heart failure secondary to ischemic or idiopathic dilated cardiomyopathy. Am J Cardiol 79(12): 1645-1650.

28. Larsson K, Hjemdahl P, Theodorsson E (1990) Acute bronchoconstriction is not a stimulus for sympatho-adrenal activation in asthmatic or healthy subjects. Eur Respir J 3(3): 273-281.

29. Casolo G, Balli E, Taddei T, Amuhasi J, Gori C (1989) Decreased spontaneous heart rate variability in congestive heart failure. Am J Cardiol 64(18): 1162-1167

30. Carter R, Hinojosa Labore C, Convertino VA (2014) Heart rate variability in patients being treated for dengue viral infection: new insights from mathematical correction of heart rate. Front Physiol 5: 46.

31. Lebech AM, Kristoffersen US, Mehlsen J, Wiinberg N, Petersen CL, et al. (2007) Autonomic dysfunction in HIV patients on antiretroviral therapy: studies of heart rate variability. Clin Physiol Funct Imaging 27(6): 363367.

32. Gadino GAM, Moraes FR, Silva GV, Accioly MF (2015) Heart rate variability in HIV-infected and AIDS. Critical Care 19(2): 6.

33. John Camm A, Bigger JT, Münster B, Cerutti S, Coumel P, et al. (1996) Heart rate variability. Standards of measurement, physiological interpretation, and clinical use. Task Force of the European Society of Cardiology and the North American Society of Pacing and Electrophysiology. Eur Heart J 17(3): 354-381.

34. Farrell TG, Bashir Y, Cripps T, Malik M, Poloniecki J,et al. (1991) Risk stratification for arrhythmic events in postinfarction patients based on heart rate variability, ambulatory electrocardiographic variables and the signal-averaged electrocardiogram. J Am Coll Cardiol 18(3): 687-697.

35. Malik M (1996) Heart rate variability - standards of measurement physiological interpretation, and clinical use. Circulation 93(5): 10431065.

36. Acharya UR, Joseph KP, Kannathal N, Lim CM, Suri JS (2006)Heart rate variability: a review. Med Bio Eng Comput 44(12): 1031-1051.

37. Costa M, Goldberger AL, Peng CK (2005) Multiscale entropy analysis of biological signals. Phys Rev E Stat Nonlin Soft Matter Phys 71(2 Pt 1): 021906

38. Huikuri HV, Makikallio TH, Peng CK, Goldberger AL, Hintze U, et al. (2000) Fractal correlation properties of R-R interval dynamics and mortality in patients with depressed left ventricular function after an acute myocardial infarction. Circulation 101(1): 47-53.

39. Kovatchev BP, Farhy LS, Cao H, Griffin MP, Lake DE, et al. (2003) Sample asymmetry analysis of heart rate characteristics with application to neonatal sepsis and systemic inflammatory response syndrome. Pediatr Res 54(6): 892-898. 
40. Griffin MP, Lake DE, Moorman JR (2005) Heart rate characteristics and laboratory tests in neonatal sepsis. Pediatrics 115(4): 937-941.

41. Moorman JR, Lake DE, Griffin MP (2006) Heart rate characteristics monitoring for neonatal sepsis. IEEE Trans Biomed Eng 53(1): 126-132.

42. Cao H, Lake DE, Griffin MP, Moorman JR (2004) Increased nonstationarity of neonatal heart rate before the clinical diagnosis of sepsis. Ann Biomed Eng 32(2): 233-244.

43. Schmidt HB, Werdan K, Muller Werdan U (2001) Autonomic dysfunction in the ICU patient. Curr Opin Crit Care 7(5): 314-322.

44. Yien HW, Hseu SS, Lee LC, Kuo TB, Lee TY, et al. (1997) Spectral analysis of systemic arterial pressure and heart rate signals as a prognostic tool for the prediction of patient outcome in the intensive care unit. Crit Care Med 25(2): 258-266.

45. Soriano F, Nogueria A, Cappi S, Lins M, Hoshino W, et al. (2004) Heart dysfunction and heart rate variablility prognoses in sepsis. Crit Care 8(1): 75.

46. Piepoli M, Garrard CS, Kontoyannis DA, Bernardi L (1995) Autonomic control of the heart and peripheral vessels in human septic shock. Intensive Care Med 21(2): 112-119.

47. Pontet J, Contreras P, Curbelo A, Medina J, Noveri S, et al. (2003) Heart rate variability as early marker of multiple organ dysfunction syndrome in septic patients. J Crit Care 18(3): 156-163.
48. Barnaby D, Ferrick K, Kaplan DT, Shah S, Bijur P, et al. (2002) Heart rate variability in emergency department patients with sepsis. Acad Emerg Med 9(7): 661-670.

49. Chen WL, Kuo CD (2007) Characteristics of heart rate variability can predict impending septic shock in emergency department patients with sepsis. Acad Emerg Med 14(5): 392-397.

50. Ahmad S, Ramsay T, Huebsch L, Flanagan S, Mc Diarmid S, et al. (2009) Continuous multiparameter heart rate variability analysis herald's onset of sepsis in adults. PLoS One 4(8): e6642.

51. Allen J (2007) Photoplethysmography and its application in clinical physiological measurement. Physiol Meas 28(3): R1-39.

52. Hernando D, Roca S, Sancho J, Alesanco Á, Bailón R (2018) Validation of the Apple Watch for Heart Rate Variability Measurements during Relax and Mental Stress in Healthy Subjects. Sensors 18(8): E2619

53. Li KHC, White FA, Tipoe T, Liu T, Wong MC, et al. (2019) The Current State of Mobile Phone Apps for Monitoring Heart Rate, Heart Rate Variability, and Atrial Fibrillation: Narrative Review. JMIR Mhealth Uhealth 7(2): e11606. 